\title{
The potential prognostic value of connexin 26 and 46 expression in neoadjuvant-treated breast cancer
}

Ivett Teleki ${ }^{1 \dagger}$, Tibor Krenacs ${ }^{1,2^{*}+}$, Marcell A Szasz ${ }^{3}$, Janina Kulka ${ }^{3}$, Barna Wichmann ${ }^{4}$, Cornelia Leo ${ }^{5}$, Barbel Papassotiropoulos ${ }^{6}$, Cosima Riemenschnitter ${ }^{7}$, Holger Moch $^{7}$ and Zsuzsanna Varga ${ }^{7}$

\begin{abstract}
Background: Several classification systems are available to assess pathological response to neoadjuvant chemotherapy in breast cancer, but reliable biomarkers to predict the efficiency of primary systemic therapy (PST) are still missing. Deregulation of gap junction channel forming connexins $(C x)$ has been implicated in carcinogenesis and tumour progression through loss of cell cycle control. In this study we correlated Cx expression and cell proliferation with disease survival and pathological response to neoadjuvant chemotherapy in breast cancers using existing classification systems.

Methods: The expression of Cx26, Cx32, Cx43, Cx46 and Ki67 was evaluated in 96 breast cancer patients prior to and after neoadjuvant chemotherapy using duplicate cores in tissue microarrays (TMA). Cx plaques of $<1 \mu m$ were detected with multilayer, multichannel fluorescence digital microscopy. Current classifications to assess residual tumour burden after primary systemic therapy included the EWGBSP, CPS-EG, Miller-Payne, Sataloff and NSABP systems.
\end{abstract}

Results: In our cohort dominated by hormone receptor (ER/PR) positive and HER2 negative cases, only the CPS-EG classification showed prognostic relevance: cases with scores $1-2$ had significantly better overall survival $(p=0.015)$ than cases with scores 3-5. Pre-chemotherapy Cx43 expression correlated positively with hormone receptor status both before and after chemotherapy and had a negative correlation with HER2 expression pre-chemotherapy. There was a positive correlation between Cx32 and HER2 expression pre-chemotherapy and between Cx32 and Ki67 expression post-chemotherapy. A negative correlation was found between post-chemotherapy Cx46 and Ki67 expression. Decreased post-chemotherapy Cx26 expression ( $<5 \%)$ statistically correlated with better overall survival $(\mathrm{p}=0.011)$. Moderate or higher $\mathrm{C} \times 46$ expression $(>20 \%)$ pre- and post-chemotherapy correlated with significantly better survival in the intermediate prognostic subgroups of EWGBSP TR2b ( $p_{\text {pre-chemo }}=0.006$; Sataloff TB ( pre-chemo $=0.005 ; p_{\text {post-chemo }}=0.029$ ) and in Miller-Payne G3 ( pre-chemo $=0.002 ; p_{\text {post-chemo }}=0.012$ ) classifications. Pre-chemotherapy, Cx46 expression was the only marker that correlated with overall survival within these subgroups.

Conclusion: Our results suggest that Cx46 and Cx26 expression in breast cancer may improve the assessment of pathological response and refine intermediate prognostic subgroups of residual tumour classifications used after neoadjuvant chemotherapy.

Keywords: Breast cancer, Connexin, Gap junction, Preoperative chemotherapy, Prognosis

\footnotetext{
* Correspondence: krenacst@gmail.com

${ }^{\dagger}$ Equal contributors

${ }^{1} 1$ st Department of Pathology \& Experimental Cancer Research, Semmelweis University, Budapest, Hungary

${ }^{2}$ MTA-SE Tumour Progression Research Group, Budapest, Hungary

Full list of author information is available at the end of the article
} 


\section{Background}

Despite of mammographic screening, early diagnosis and available targeted therapy, breast cancer is still one of the most frequent cause of tumour related death of women in the western world [1]. Molecular subtyping and related new therapeutic approaches require diagnostic screening for at least the hormone receptors (ER, PR) and HER2 overexpression/gene amplification as predictive and prognostic markers [2]. To date several classifications have been developed to assess pathologic response to neoadjuvant chemotherapy. Most systems are based on the amount of residual tumour in the breast and the axilla [3-7]. The recently described CPS-EG score combines clinical and pathological stage with nuclear grade and hormone receptor status $[8,9]$. However, reliable biomarkers to prognosticate response to primary systemic therapy (PST) are still missing.

The gap junction forming connexins $(\mathrm{Cx})$ mediate direct cell-cell communication and their dysfunction can contribute to carcinogenesis and tumour progression in a wide range of neoplasias including breast cancer [10]. Connexins form gap junction channels that allow the regulated transport of $<1.5 \mathrm{kDa}$ molecules, including secondary messengers $\left(\mathrm{Ca}^{2+}, \mathrm{cAMP}, \mathrm{IP} 3\right)$, metabolites (ATP, $\mathrm{NAD}^{+}$, small peptides and nucleotides) between adjacent cells [11] to coordinate functions within cell compartments [12]. They are abundant in all human solid tissues and more than one isotype of the 21 cloned human isotypes - are found in most cell types [13]. Connexins play crucial roles in cell homeostasis including of regulation of cell growth, proliferation and apoptosis, either as gap junctions, hemichannels or through protein-protein interactions $[14,15]$. Therefore, functional Cx can be localized both to the cell membrane and the cytoplasm [16]. In normal mammary epithelium, Cx43 has been found in the myoepithelial cells and Cx26 in the luminal epithelium [17].

Connexins and gap junctions have been linked to carcinogenesis through aberrant expression and functions [18]. They show tumour stage dependent expression and may play opposing roles during breast cancer progression [19]. Connexin expression is usually down-regulated upon malignant transformation, but it can also mediate tumour cell endothelial interactions during tumour diapedesis [20]. Cx43 and Cx26 can be involved in tumour suppression in early stage breast cancer, but these connexins and Cx32 may also support metastatic tumour colonization by upregulation in the lymph nodes [21-23]. Moreover, new connexin isotypes such as Cx46 can appear in breast cancer, which may assist MCF-7 breast cancer cells in adapting to hypoxia [24].

The aim of this study was to correlate connexin expression and cell proliferation with clinicopathological parameters (stage, ER, PR and HER2) and prognosis in breast cancer patients treated with PST. Of the 15 of 21 connexin isotypes (Cx23, Cx26, Cx30, Cx30.2, Cx30.3, Cx31, Cx31.1, Cx32, Сx36, Сx37, Cx43, Cx45, Cx46, Cx50 and Cx62) tested in a pilot study Cx26, Cx32, $\mathrm{Cx} 43$ and $\mathrm{Cx} 46$ were detected reliably and widespread in breast cancers. These isotypes were screened together with Ki67 expression prior to- (pre-chemo) and after neoadjuvant chemotherapy (post-chemo) using wholeslide immunofluorescence digital microscopy in 96 breast cancers included in tissue microarrays (TMA). Results were correlated with subgroups of the current classifications including the CPS-EG score (Clinical Pathological Stage combined with Estrogen receptor status and Grade by M.D. Anderson Cancer Center, Texas), NSABP (National Surgical Adjuvant Breast and Bowel Project), Miller-Payne 'G', Sataloff 'T' and EWGBSP 'TR' (European Working Group for Breast Screening Pathology) systems [3-9]. Our results suggest the potential prognostic value of $\mathrm{Cx}$ detection in neoadjuvant treated breast cancer. Cases with reduced Cx26 expression postchemotherapy and those with moderate to high $\mathrm{Cx} 46$ expression both pre- and post-chemotherapy showed significantly improved survival rates particularly in the intermediate subgroups of current classification systems.

\section{Methods}

\section{Patient cohort}

96 patients with breast cancer, diagnosed either in core biopsies or fine needle aspiration biopsy (FNAB) and then treated with neoadjuvant chemotherapy were selected consecutively between 1998 and 2009 from the archives of the Institute of Surgical Pathology, University Hospital Zurich, Switzerland. Chemotherapy regimens included Docetaxel $75 \mathrm{mg} / \mathrm{m}^{2}$; Epirubicin $90 \mathrm{mg} / \mathrm{m}^{2}$; Cyclophosphamide $500 \mathrm{mg} / \mathrm{m}^{2}$; Doxorubicin $50 \mathrm{mg} / \mathrm{m}^{2}$; Vinorelbine $30 \mathrm{mg} / \mathrm{m}^{2}$; Fluorouracil $500 \mathrm{mg} / \mathrm{m}^{2}$; and also Trastuzumab $\left(250 \mathrm{mg} / \mathrm{m}^{2}\right)$. The exact preoperative chemotherapy schedules were available in 73 patients (Docetaxel/ Epirubicin n=24, Epirubicin/Cyclophosphamide /Fluorouracil $\mathrm{n}=23$, Docetaxel/Epirubicin/Cyclophosphamide $\mathrm{n}=14$, Docetaxel/Trastuzumab $n=7$, Vinorelbine/Trastuzumab $n=5$ ). Chemotherapy schedules were administered in 2 to 6 cycles.

Formalin-fixed, paraffin-embedded (FFPE) tumour blocks from preoperative core biopsies and from the corresponding postoperative tissues were available in 64 patients. Core biopsies prior to neoadjuvant chemotherapy without surgical specimens after chemotherapy were available in 17 patients. Surgical specimens following the chemotherapy without previous core biopsies (diagnosed with FNAB) were available in 15 patients. Clinicopathological and followup data ( 2 to 10 years) on 96 patients could be retrieved from the pathological and clinical files as summarized in Table 1. Histologically or cytologically 75 tumours (78\%) were diagnosed as invasive ductal carcinoma, 18 (19\%) as invasive 
Table 1 Clinicopathological features of breast cancer patients studied

\begin{tabular}{|c|c|c|c|c|}
\hline $\mathrm{n}=96$ & \multicolumn{2}{|c|}{ Prior to chemotherapy } & \multicolumn{2}{|c|}{ After chemotherapy } \\
\hline \multirow[t]{12}{*}{ Tumour size } & \multicolumn{2}{|c|}{$1,5-13 \mathrm{~cm}$} & \multicolumn{2}{|c|}{$0,3-14 \mathrm{~cm}$} \\
\hline & cT1 & $1(1 \%)$ & урт0 & $6(6 \%)$ \\
\hline & $-a$ & & ypT1 & $20(21 \%)$ \\
\hline & $-b$ & & $-a$ & 2 \\
\hline & $-c$ & 1 & $-b$ & 11 \\
\hline & $\mathrm{cT} 2$ & $25(26 \%)$ & $-c$ & 7 \\
\hline & cT3 & $22(23 \%)$ & ypT2 & $31(32 \%)$ \\
\hline & cT4 & $41(43 \%)$ & урТ3 & $24(25 \%)$ \\
\hline & $-b$ & 19 & урт4 & $7(7 \%)$ \\
\hline & $-d$ & 22 & $-b$ & 6 \\
\hline & NA & $7(7 \%)$ & $-d$ & 1 \\
\hline & & & No surgery & $8(9 \%)$ \\
\hline \multirow[t]{6}{*}{ Lymph node status } & $\mathrm{cNO}$ & $10(11 \%)$ & $\mathrm{pNO}$ & $25(26 \%)$ \\
\hline & $\mathrm{cN} 1$ & $62(64 \%)$ & $\mathrm{pN} 1$ & $27(28 \%)$ \\
\hline & $\mathrm{cN} 2$ & & $\mathrm{pN} 2$ & $12(12 \%)$ \\
\hline & $\mathrm{cN} 3$ & $3(3 \%)$ & pN3 & $14(15 \%)$ \\
\hline & & & No surgery & $8(9 \%)$ \\
\hline & NA & $21(22 \%)$ & NA & $10(10 \%)$ \\
\hline \multirow[t]{4}{*}{ ER status } & Positive & $68(71 \%)$ & Positive & $59(62 \%)$ \\
\hline & Negative & $25(26 \%)$ & Negative & $16(17 \%)$ \\
\hline & NA & $3(3 \%)$ & NA & $21(21 \%)$ \\
\hline & & \multicolumn{3}{|c|}{ урт0, no surgery } \\
\hline \multirow[t]{4}{*}{ PR status } & Positive & $59(62 \%)$ & Positive & 46 (48\%) \\
\hline & Negative & 34 (35\%) & Negative & 29 (31\%) \\
\hline & NA & $3(3 \%)$ & NA & $21(21 \%)$ \\
\hline & & \multicolumn{3}{|c|}{ урT0, no surgery } \\
\hline \multirow[t]{4}{*}{ HER2 status } & Positive & $28(29 \%)$ & Positive & $18(19 \%)$ \\
\hline & Negative & $65(68 \%)$ & Negative & $57(60 \%)$ \\
\hline & NA & $3(3 \%)$ & NA & $21(21 \%)$ \\
\hline & & \multicolumn{3}{|c|}{ урт0, no surgery } \\
\hline \multirow[t]{13}{*}{ Ki67 status } & 0 & $2(2 \%)$ & 0 & $22(28 \%)$ \\
\hline & $1(0-1 \%)$ & $12(15 \%)$ & 1 & $21(27 \%)$ \\
\hline & $2(1-5 \%)$ & 19 (23\%) & 2 & $11(14 \%)$ \\
\hline & $3(5-10 \%)$ & $13(16 \%)$ & 3 & $3(4 \%)$ \\
\hline & $4(10-15 \%)$ & $6(7 \%)$ & 4 & $1(1 \%)$ \\
\hline & $5(15-20 \%)$ & $4(5 \%)$ & 5 & $6(8 \%)$ \\
\hline & $6(20-33 \%)$ & $2(2 \%)$ & 6 & $1(1 \%)$ \\
\hline & 7 (33-50\%) & $4(5 \%)$ & 7 & $2(3 \%)$ \\
\hline & $8(50-66 \%)$ & $1(1 \%)$ & 8 & $0(0 \%)$ \\
\hline & $9(66-80 \%)$ & $3(4 \%)$ & 9 & $3(4 \%)$ \\
\hline & 10 (80-100\%) & $1(1 \%)$ & 10 & $2(3 \%)$ \\
\hline & NA & $14(17 \%)$ & NA & $7(9 \%)$ \\
\hline & $\sum$ & $64+17(100 \%)$ & $\sum$ & $64+15(100 \%)$ \\
\hline
\end{tabular}


lobular carcinoma, 2 cases were metaplastic squamous cell carcinomas (2\%) and one case was a small cell carcinoma. Patient's age ranged from 30 to 74 years (mean age: 52 years). Eight of 96 patients (9\%) had multifocal tumours on imaging. Histological grading according to the modified BloomRichardson score could be done in 81 cases: 38 cases (40\%) were poorly differentiated (grade 3) carcinomas, 42 (44\%) moderately differentiated (grade 2), one case (1\%) was well differentiated (grade 1), 15 cases (15\%) had only small amount of tumour tissue insufficient for correct grading on the core [25].

Mastectomy was performed in 60 patients (62\%) and segmentectomy in 28 patients (29\%). Breast surgery was completed with axillary dissection in all but 5 of these patients. In 8 patients (9\%) either no surgery was performed or we had no records in our files. Fifteen of 96 patients (16\%) had multifocal tumours at the time of surgery. Tumour cellularity was estimated as the percentage of residual tumour cells distributed in the tumour bed area [6]. The histological subtypes were identical to those of the preoperative biopsies. The frequency of ER/PR and HER2 positive cases in our cohort roughly reflected that of the general breast cancer population.

The study and the construction of TMA was approved by the Ethical Committee of the Canton Zurich (KEKZH NR: 2009-0065) and also by the Internal Review Board of the Institute of Surgical Pathology.

\section{Detection of hormone receptors and HER2}

Estrogen receptor (ER, clone 6F11) and progesterone receptor (PR, clone 1A6) expression was determined using the iVIEW DAB detection kit in Ventana Benchmark (all from Ventana, Basel, Switzerland) immunostainer following heat induced epitope retrieval in $\mathrm{CC} 1$ solution. Cases of $>1 \%$ nuclear positive tumour cells were considered as positive [25].

HER2 status was defined according to the initial and the modified ASCO criteria using immunohistochemistry and/or fluorescence in situ hybridization (FISH) (19982004 IHC and FISH, 2004-2009 only FISH) [26].

Between 1998-2004 the Pathway ${ }^{\text {TM }}$ HER2 (clone CB11) FDA approved kit (Ventana) was used for automated immunostaining as described above. Cases with tumour cells of $>10 \%$ strong and complete membrane staining were considered $3+$; and those with $>10 \%$ moderate, but complete membrane staining as $2+$ requiring additional FISH testing. During the 1998-2009 period HER2 gene amplification was tested using the dual colour FISH kit of PathVision (Vysis, Abbott AG, Baar, Switzerland) according to the manufacturer's protocol.

\section{Tissue microarray construction}

Hematoxylin-eosin (H\&E) stained sections of all FFPE tumours were re-evaluated by one pathologist (Z.V.) for suitability for TMA, which were prepared as described earlier containing duplicate cores from each patient's samples $[27,28]$. Core biopsies from 81 patients prior to chemotherapy (64 matched surgical and core biopsy; and 17 core biopsy only) and tumour samples from 79 patients after chemotherapy (64 paired as above and 15 surgical) were arrayed into two TMA blocks.

\section{Immunofluorescence detection of connexins}

TMA slides of $4 \mu \mathrm{m}$ thick were dewaxed in xylene and rehydrated through ethanol series. Antigen unmasking was done in a 0.1 M Tris 0.01 M EDTA buffer ( $\mathrm{pH} 9.0$ ) using an electric pressure cooker (Avair, Biofa, Veszprem, Hungary) for $20 \mathrm{~min}$ at $\sim 105^{\circ} \mathrm{C}$ followed by $10 \mathrm{sec}$ digestion in $0,25 \%$ Gibco trypsin phenol red (1:50; Life Technologies, Carlsbad, CA, Ref: 25050-014). After a protein blocking step for $20 \mathrm{~min}$ the slides were incubated overnight using rabbit anti-mouse Cx26 (1:4000, AB8143, Millipore, Billerica, MA) or anti-human Cx32 (1:30, HPA010663, Sigma-Aldrich, St Luis, MO), Cx43 (1:100, \#3512, Cell Signaling, Beverly, MA) or Cx46 (1:100, SAB13005557, Sigma-Aldrich) antibodies. All animal specific connexin antibodies used highly cross-react with the relevant human connexins [29]. TMA slides were simultaneously also stained for Ki-67 protein using the mouse anti-human Mib-1 antibody (1:2 ready-to-use, IR626, Dako, Glostrup, Denmark).

Fluorochrome-labelled secondary antibodies, Alexa Fluor 546 (red) goat anti-rabbit IgG (A11035) and Alexa Fluor 488 (green) goat anti-mouse IgG (A11001) were applied in 1:200 for 90 min. Finally, Hoechst (1:1000, B2883, blue) was used for nuclear staining (all from InvitrogenLife technologies, Eugene, OR), for 60 sec. All incubations were done in a humid chamber at room temperature and slides were washed between the steps using $0.1 \mathrm{M}$ TBS (Tris-buffered saline) $\mathrm{pH} 7.4$ for $2 \times 5 \mathrm{~min}$. Immunostained slides were digitalized using Pannoramic Scan (3DHISTECH Ltd., Budapest, Hungary). The multilayer, multichannel fluorescence digitalization of TMA slides resulted in permanent fluorescence samples and allowed accurate analysis of connexin plaques of frequently $<1 \mu \mathrm{m}$ size within the whole section thickness without the risk of false negativity due to fading or lack of focus depth resolution.

\section{Scoring of connexin expression and cell proliferation}

Immunoreactions for $\mathrm{Cx}$-s were evaluated by two independent assessors using a 4-scale scoring system with the TMA module software [30] (3DHISTECH) by considering the frequency of positively stained cells as follows: score 0: $<5 \%$; score 1: $5-20 \%$; score 2: $20-60 \%$; score 3: $>60 \%)$. Positive signals of all assessed connexin subtypes were localized in the plasma membrane in the normal breast epithelial cells. In breast cancer samples, 
positive reaction was mainly seen in the cytoplasm of the invasive tumour cells. Both of these signal types were considered upon scoring. Stromal, smooth muscle and endothelial cells, adipocytes, skin, keratinocytes and normal mammary epithelium served as endogenous positive controls. Ki-67 reaction was assessed on a linear 0-10 scale (score 0: 0, 1: 0-1\%, 2: 1-5\%, 3: 5-10\%, 4: 10-15\%, 5:15-20\%, 6: $20-33 \%, 7: 33-50 \%, 8: 50-66 \%$, 9: 66-80\%, 10: 80-100\%) considering the frequency of positive tumour cells (Table 1 ). In case of tumour heterogeneity the higher score was considered for statistics.

\section{Assessing tumour response after neoadjuvant chemotherapy using histopathological and clinical classification systems}

Five current pathological classification systems were applied retrospectively to assess pathologic response after neoadjuvant chemotherapy. Four of these systems
NSABP, Miller-Payne Grade, Sataloff T and EWGBSPTR analyse the extent of residual tumour tissue (none, in situ or invasive). These could be applied retrospectively in 89 cases. The CPS-EG score classification combines clinical and pathological stages with nuclear grading and hormone receptor expression, which could be used in 55 cases. Details of pathological response and definition of classification systems are summarized in Table 2.

\section{Statistical analysis}

SPSS 15.0 software was used for statistical comparisons (SPSS, Inc., Chicago, IL, USA). Categorical data were analysed using chi-square test. Spearman rank correlation was used to correlate connexin scores and clinicopathological parameters (stage and grade, hormone receptor status, HER2 status). Overall survival was calculated using Kaplan-Meier method with assessment of statistical significance by log-rank test. For multivariate analysis Cox-regression analysis with 95\%

Table 2 Definition of classification systems and distribution of the enrolled cases

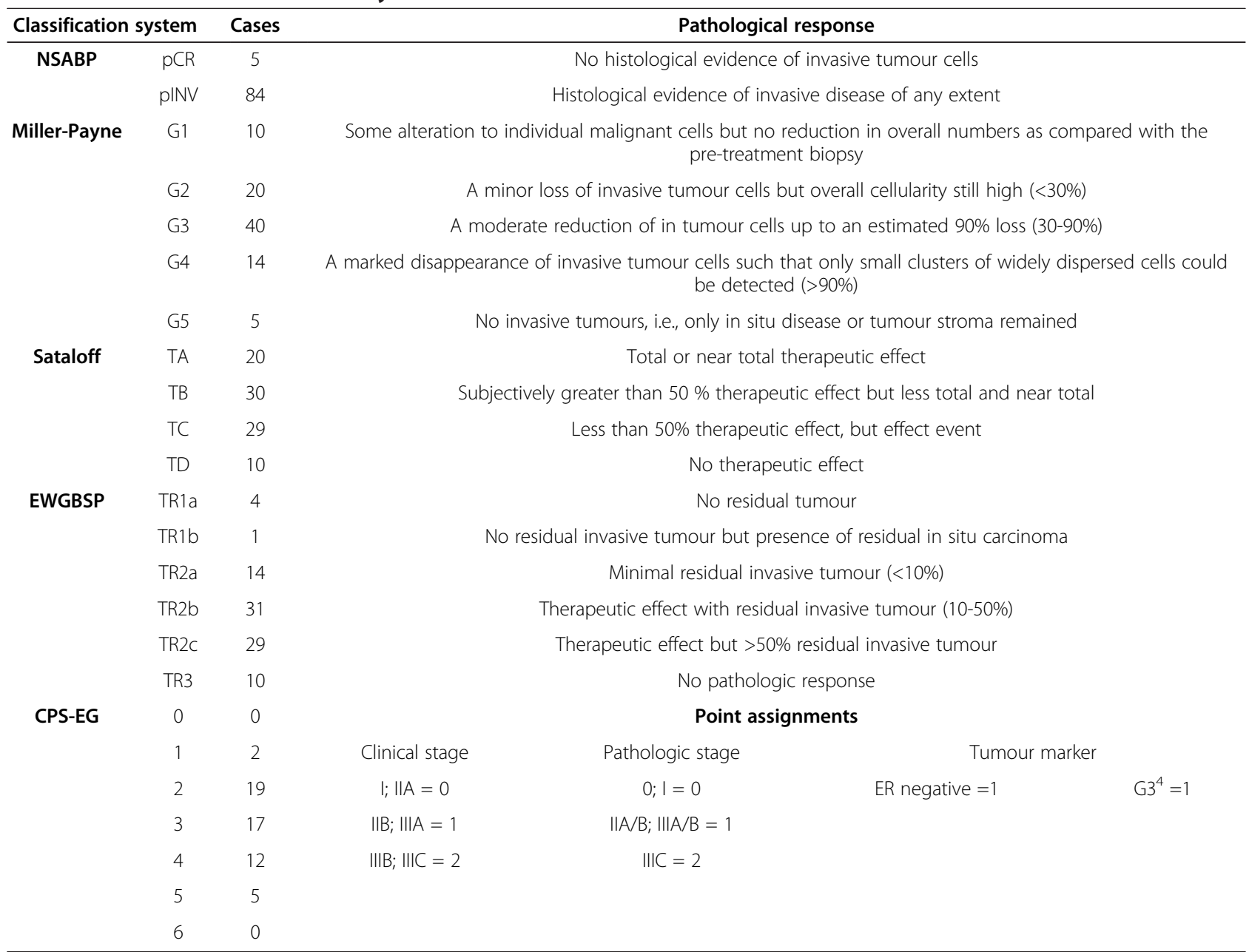


confidence intervals was performed by including Cx26, Cx46, hormone receptor status, HER2 status, prechemo tumour size, proliferation, pathological response, stage and grade which did not correlate directly with each other. Results were considered statistically significant at $\mathrm{p}$-values of $<0.05$. Bonferroni correction was not applied.

\section{Results}

\section{Connexin expression before and after neoadjuvant} chemotherapy

Connexin expression upon chemotherapy showed dynamic but differential changes related to the isotypes which is also revealed in details for individual cases in (Figure 1). Examples of related connexin immunofluorescence are seen in Figure 2. In the whole patient cohort, the number of Cx26 expressing tumours and the percentage of Cx26 positive tumour cells in individual tumours significantly decreased after chemotherapy. The number of cases scoring 0 increased from $3 \%$ to $25 \%$ ( $p<0.001$ ), while the number of cases scoring 3 decreased from $32 \%$ to $10 \%$ ( $\mathrm{p}<0.006$ ). The frequency of intermediate scores (1 and 2) did not change during therapy. Cx32 expression was significantly reduced after chemotherapy. The frequency of cases scoring 0 (negative) increased from $10 \%$ to $49 \%$ ( $\mathrm{p}<0.004$ ) while of those scoring 1 (weak positive) decreased from $35 \%$ to $17 \%$ ( $\mathrm{p}<0.043$ ). The frequency either of Cx43 or Cx46 positive cases did not differ significantly before and after chemotherapy, though, the number of $3+\mathrm{Cx} 46$ positive cases showed a tendency of increase post-chemotherapy. Of 64 matched samples 50 (for Cx43 51 cases) could be assessed reliably. Cx26 and Cx32 were mainly decreased, $\mathrm{Cx} 43$ showed the least change while Cx46 levels showed an increasing tendency upon chemotherapy (Figure 1B-E).

\section{Correlation of connexin expression with clinicopathological parameters before and after neoadjuvant chemotherapy}

Correlations between connexin expression, cell proliferation and clinicopathological features are summarized in Table 3.

Cx26 expression in cases of $<5 \%$ positive tumour cells (score 0 ) post-chemo statistically correlated with improved overall survival $(\mathrm{p}=0.011)$ compared to those of $>5 \% \mathrm{Cx} 26$ positive tumour cells (scores 1-3) (Figure 3). However, Cx26 expression did not show correlation with other clinicopathological parameters when using the Spearman rank $(\rho)$ test. At the same time, Cx32 expression had a positive correlation with HER2 status both pre-chemo $(\rho=0.31)$ and a negative correlation to pathological tumour stage (pT) $(\rho=-0.29)$. Moreover, post-chemo Cx32 and Ki67 expression showed a positive correlation $(\rho=0.46)$. Pre-chemo Cx43 expression positively correlated with ER and PR expression both pre- and post-chemo and with the clinical tumour stage (cT) $(\rho=0.29-0.36)$ and had a negative correlation with HER2 status pre-chemo $(\rho=-0.27)$. Cx46 expression pre- and post-chemo had a positive statistical correlation with nodal status $(\mathrm{pN})$ ( $\rho=0.39$ and 0.32 ) and pre-chemo Cx46 and Ki67 expression negatively correlated with each other $(\rho=-0.29)$. A negative correlation was found between ER and Ki67 expression both pre- and post-chemo $(\rho=-0.35--0.46)$ and between PR and Ki67 expression pre-chemo ( $\rho=-0.45$; -0 .52). Furthermore, Ki67 expression had a positive correlation with HER2 status both post-chemo $(\rho=0.26)$ and a positive correlation was detected between pre-and postchemo Ki67 scores $(\rho=0.46)$.

\section{Correlation between the defined clinicopathological response groups and overall survival}

Primary breast cancer cases were classified according NSABP, Miller-Payne, Sataloff, EWGBSP, CPS-EG systems. Only the CPS-EG classification showed correlation with overall survival: cases with scores 1 and 2 had significantly better survival rate $(p=0.015)$ than those scoring 3, 4 and 5 (Figure 4).

\section{Overlap among subgroups of the classification systems}

We also tested how subgroups of the classification systems overlap in our patient cohort (Figure 5A). In general, only few cases fell into the subgroups with edge categories such as EWGBSP TR1a-b and TR3; CPS-EG 1 and 5; Sataloff TA and TD; or Miller-Payne GI and G5. These included the particularly responsive and the barely/non-responsive tumours which highly overlapped among classifications. The rest of cases were heterogeneously sorted into the intermediate categories including EWGBSP TR2a-TR2c; CPS-EG 2-4; Sataloff TB and TC; and the Miller-Payne G2-G4. The NSABP is a binary system without intermediate subgroups based on the presence or lack of residual tumours. Five cases fell into the pCR group of NSABP, the rest (84 cases) were assigned to the pINV group.

\section{Prognostic potential of connexin expression on residual tumour classifications}

We analysed Cx26, Cx32, Cx43 and Cx46 expression within each classification subgroup in correlation with overall survival. Due to the Gaussian distribution of cases within the used classifications and the high agreement either within the good or the bad prognostic subgroups at the edge categories we focused on the intermediate subgroups.

Cases with $2+$ and $3+$ pre-chemo Cx46 expression $(>20 \%)$ had significantly better survival rates in EWGBSP TR2b ( $\mathrm{p}=0.006)$, Sataloff TB (0.005) and Miller-Payne G3 ( $\mathrm{p}=0.002)$ subgroups, than those scoring 0 or $1+(<20 \%)$ for $\mathrm{Cx} 46$. This correlation was almost the same for post-chemo $\mathrm{Cx} 46$ expression. In the 


\begin{tabular}{|c|c|c|c|}
\hline A & $\begin{array}{c}\text { Before } \\
\text { chemotherapy } \\
(n=81)\end{array}$ & $\begin{array}{c}\text { After } \\
\text { Chemotherapy } \\
(n=79)\end{array}$ & p-values \\
\hline \multicolumn{4}{|c|}{ Connexin 26} \\
\hline Score 0 & $2(3 \%)$ & $20(25 \%)$ & $p<0.001$ \\
\hline Score 1 & $17(21 \%)$ & $17(22 \%)$ & ns \\
\hline Score 2 & $24(30 \%)$ & $24(31 \%)$ & ns \\
\hline Score 3 & $26(32 \%)$ & $8(10 \%)$ & $p<0.006$ \\
\hline NA & $12(14 \%)$ & $10(12 \%)$ & \\
\hline \multicolumn{4}{|c|}{ Connexin 32} \\
\hline Score 0 & $15(19 \%)$ & $39(49 \%)$ & $p<0.004$ \\
\hline Score 1 & $28(35 \%)$ & $13(17 \%)$ & $p<0.043$ \\
\hline Score 2 & $17(21 \%)$ & $11(14 \%)$ & ns \\
\hline Score 3 & $7(8 \%)$ & $7(9 \%)$ & ns \\
\hline NA & $14(17 \%)$ & $9(11 \%)$ & \\
\hline \multicolumn{4}{|c|}{ Connexin 43} \\
\hline Score 0 & $42(51 \%)$ & $44(56 \%)$ & ns \\
\hline Score 1 & $9(11 \%)$ & $16(20 \%)$ & ns \\
\hline Score 2 & $8(10 \%)$ & $7(9 \%)$ & ns \\
\hline Score 3 & $11(14 \%)$ & $4(5 \%)$ & ns \\
\hline NA & $11(14 \%)$ & $8(10 \%)$ & \\
\hline \multicolumn{4}{|c|}{ Connexin 46} \\
\hline Score 0 & $18(22 \%)$ & $19(24 \%)$ & ns \\
\hline Score 1 & $19(23 \%)$ & $9(11 \%)$ & ns \\
\hline Score 2 & $16(20 \%)$ & $12(15 \%)$ & ns \\
\hline Score 3 & $16(20 \%)$ & $27(35 \%)$ & ns \\
\hline NA & $12(15 \%)$ & $12(15 \%)$ & \\
\hline
\end{tabular}
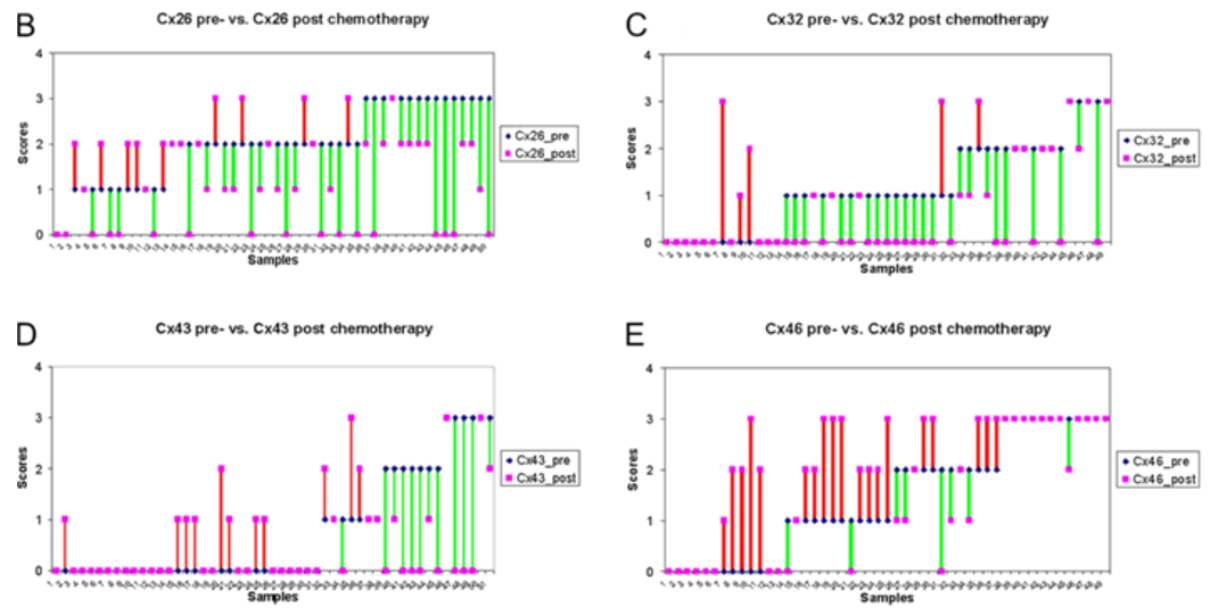

Figure 1 Expression of connexin isotypes in breast cancers before and after neoadjuvant chemotherapy (A). Dynamic changes in connexin isotype expression in the 50 (in Cx43, 51) matched breast cancer samples upon treatment (B-E). Cx26 (B) and Cx32 (C) levels mostly decreased, $\mathbf{C x 4 3}$ (D) levels revealed the least change, while $\mathbf{C x 4 6}$ (E) levels were mainly increased. Green lines represent decrease, red lines highlight increase. Violet symbols alone represent no change in expression.

Sataloff TB group the tumours with $2+$ and $3+$ Cx46 expression $(>20 \%)$ had significantly better survival rates $(\mathrm{p}=0.029)$ than those scoring 0 and $1+(<20 \%)$. In the Miller-Payne G3 category the tumours with 1-3+ Cx46 expression $(>5 \%)$ showed significantly better survival $(\mathrm{p}=0.012)$ than those scoring $0(<5 \%)$. In the EWGBSP TR2b group the tumours with $2+$ and $3+\mathrm{Cx} 46$ scores showed nearly significant $(\mathrm{p}=0.059)$ better survival compared to cases scoring 0 and $1+$ (Figure 6).

Cases with $<5 \%$ Cx26 positive cells (score 0 ) had significantly better survival rates in NSABP pINV tumours than those with $>5 \%$ (score $1+, 2+, 3+$ ) Cx26 positive cells $(\mathrm{p}=0.013)$. Intermediate categories in the other subgroups also showed the same tendency but without statistical significance including EWGBSP TR2b ( $\mathrm{p}=0.08)$, Sataloff TB ( $\mathrm{p}=0.092)$, Miller-Payne 'G2' $(\mathrm{p}=0.053)$ and CPS-EG 4 $(\mathrm{p}=0.06)$.

\section{Prognostic potential of $\mathrm{C} \times 26$ and $\mathrm{Cx} 46$ in the whole patient cohort and in the subgroups}

Using Kaplan-Meier estimates supported by log-rank test the expression of post-chemo Cx26, ER and PR status, pre- and post-chemo $\mathrm{Cx} 46$ as well as the CPS-EG classification showed statistical correlation with overall survival. Those features which were not correlated with each other among these when using the chi-square test were involved in multivariate Cox-regression analysis (Table 4). HER2 status, proliferation, grade, stage, pre- 


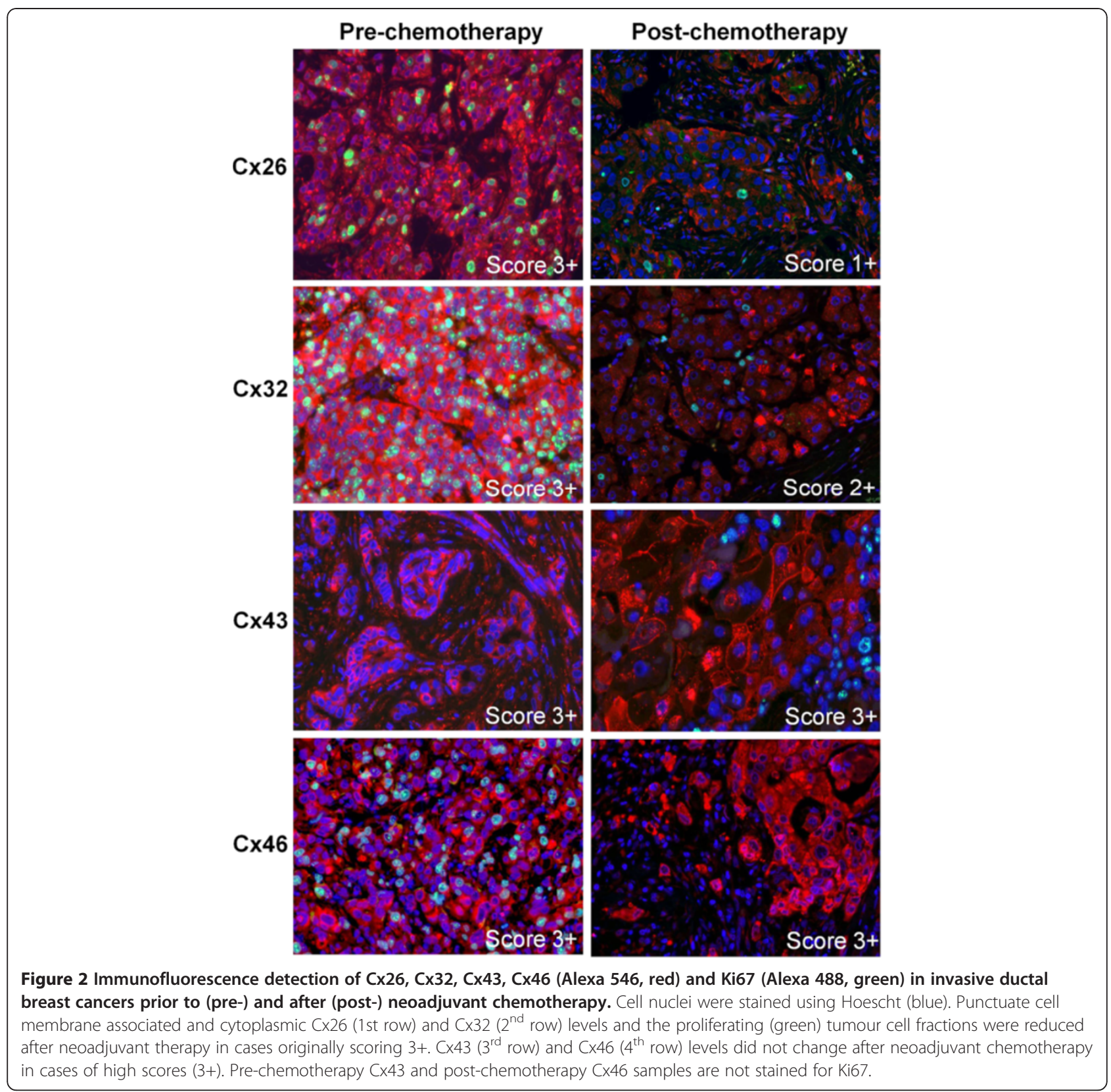

chemo tumour size, pathological response (tumour cellularity) did not correlate with overall survival, therefore, they were omitted from further analysis.

In the whole patient cohort, cases with hormone receptor positivity after chemotherapy had a significantly improved overall survival $\left(\mathrm{p}_{\mathrm{ER}}=0.012, \mathrm{p}_{\mathrm{PR}}=0.002\right)$. Postchemo PR and ER showed a better independent prognostic value according to the multivariate analysis than post-chemo Cx26 levels and CPS-EG which latter two were statistically not significant (Table 4).

In the intermediate prognostic categories (EWGBSP TR2b, Sataloff TB and Miller-Payne G3) none of the tested parameters correlated with overall survival pre- chemo except $\mathrm{Cx} 46_{\text {pre }}$, which thus proved to be the only potential prognostic factor. Post-chemo, only PR positive cases had a significantly better overall survival in the EWGBSP TR2b ( $\mathrm{p}=0.03)$, Sataloff TB $(\mathrm{p}=0.05)$ and

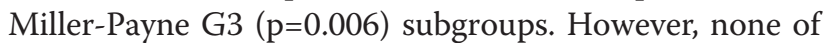
these markers proved to be significant independent prognostic factors in multivariate Cox-regression analysis (Table 4).

\section{Discussion}

Neoadjuvant chemotherapy is now considered as one of the standard options in the treatment of locally advanced and also for primary operable breast cancers 
Table 3 Correlations of connexin and Ki67 proliferation marker expression with clinicipathological parameters

\begin{tabular}{|c|c|c|c|c|c|c|c|c|c|c|}
\hline & \multicolumn{2}{|c|}{ Cx43 } & \multicolumn{2}{|c|}{ Cx26 } & \multicolumn{2}{|c|}{ Cx32 } & \multicolumn{2}{|c|}{ Cx46 } & \multicolumn{2}{|c|}{ Ki67 } \\
\hline & pre & post & pre & post & pre & post & pre & post & pre & post \\
\hline ER pre & 0.30 & $x$ & ns & $x$ & ns & $x$ & ns & $x$ & -0.46 & -0.35 \\
\hline ER post & 0.36 & ns & ns & ns & ns & ns & ns & ns & -0.40 & -0.38 \\
\hline PR pre & 0.34 & $x$ & ns & $x$ & ns & $x$ & ns & $x$ & -0.45 & $x$ \\
\hline PR post & 0.33 & ns & ns & ns & ns & ns & ns & ns & -0.52 & ns \\
\hline HER2 pre & -0.27 & $x$ & ns & $x$ & 0.31 & $x$ & ns & $x$ & ns & $x$ \\
\hline HER2 post & ns & ns & ns & ns & ns & ns & ns & ns & ns & 0.26 \\
\hline cT pre & 0.29 & $x$ & ns & $x$ & ns & $x$ & ns & $x$ & ns & $x$ \\
\hline cN pre & ns & $x$ & ns & $x$ & ns & $x$ & ns & $x$ & ns & $x$ \\
\hline pT post & ns & ns & ns & ns & -0.29 & ns & ns & ns & ns & ns \\
\hline pN post & ns & ns & ns & ns & ns & ns & 0.39 & 0.32 & ns & ns \\
\hline grade pre & ns & $x$ & ns & $x$ & ns & $x$ & ns & $x$ & ns & ns \\
\hline Ki67 pre & ns & $x$ & ns & $x$ & ns & $x$ & -0.29 & $x$ & $x$ & 0.46 \\
\hline Ki67 post & ns & ns & ns & ns & ns & 0.46 & ns & ns & 0.46 & $x$ \\
\hline
\end{tabular}

ns: not significant; $x$ : not relevant; pre: prior to chemotherapy; post: after chemotherapy.

[5-8]. Morphological classification systems have been established to assess the pathological response and prognosis after systemic primary therapy. These consider pathological features such as the percentage of residual tumour cells, the presence or absence of in situ component with or without considering nodal status [3,5-8]. Reliable biomarkers to assess response to primary systemic therapy are not yet available. Connexins may function as gap junctions or hemichannels in the cell membranes or through intra-cytoplasmic protein interactions and play fundamental roles in cell homeostasis including cell cycle control $[11,16]$. In this study we analysed the expression of four connexin isotypes Cx26, Cx32, Cx43 and Cx46, and Ki67 for cell

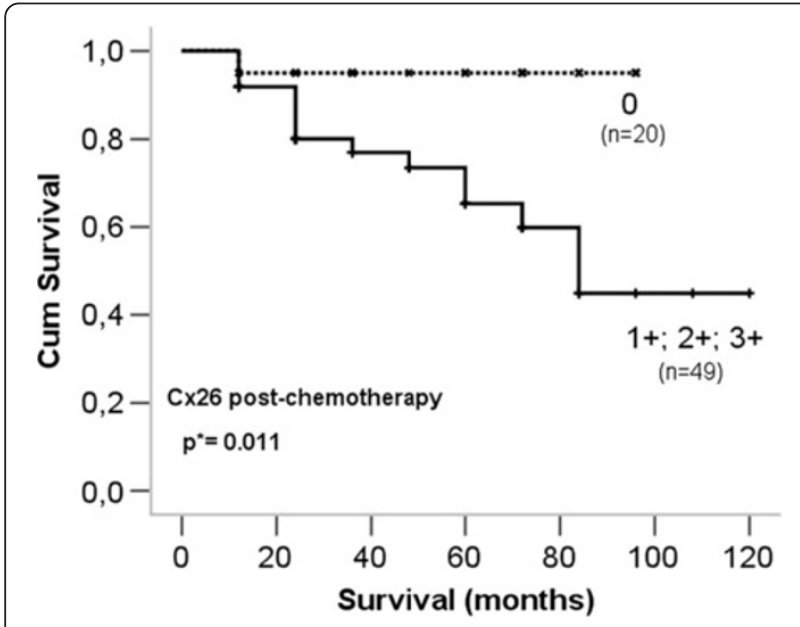

Figure 3 Kaplan-Meier plot of overall survival based on Cx26 expression after chemotherapy. Log-rank test reveals significant association ( $p=0.011$ ) between reduced $\mathrm{C} \times 26$ expression $(0 ;<5 \%)$ and improved disease outcome. proliferation prior to and after neoadjuvant chemotherapy in breast cancer in correlation with clinicopathological parameters, overall survival and pathological response based on current classification systems.

Our main results suggest that increased Cx46 positivity either pre- or post-chemotherapy and reduced Cx26 expression post-chemotherapy may separate prognostically more favourable subgroups within the intermediate categories of the classifications including Miller-Payne G2-3, Sataloff TB, EWGBSP TR2b and CPS-EG 4 (Figure 5B). In addition, pre-chemotherapy only Cx46 expression appeared to correlate with overall survival. Others have also observed that biomarkers such as PR, HER2, grade, or tumour size did not necessarily correlate with prognosis either before or after chemotherapy [31]. In agreement with those who showed elevated Cx26 expression to contribute to carcinogenesis in pancreatic and prostate carcinoma [32,33] we also found a significantly reduced Cx26 expression after chemotherapy in association with better overall survival in breast cancer $(\mathrm{p}=0.011)$. Moreover, decreased Cx26 expression after chemotherapy could be more promising for prognosis than classical features such as HER2 expression, grade, Ki67 index according to our results. Interestingly, loss of Cx26 expression has also been implicated in reduced survival in primary gastric and colorectal carcinomas, suggesting an organ specific biological significance of Cx26 [34,35].

The role of connexins in breast cancer development, progression and metastatic growth has been the subject of only few previous studies all focusing on primary breast cancers [21-23,36]. The transitional loss of Cx43 has been reported where re-expression of $\mathrm{Cx} 43$ might sensitize breast cancer cell lines for chemotherapeutic 


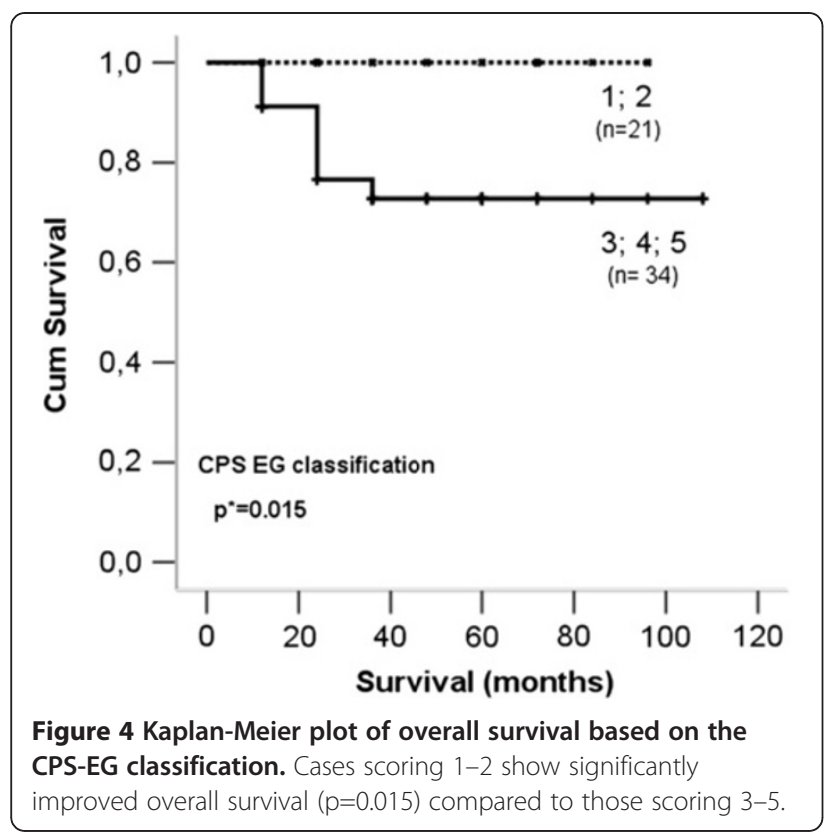

agents [36]. Primary breast cancers exhibiting elevated expression of Cx26, Cx32 and $\mathrm{Cx} 43$ have been found more often in node positive than node-negative breast cancers [21,22]. Although, Cx46 has been implicated in protecting MCF-7 breast cancer cells from hypoxia, apart from our study, there has been no data revealed on the prognostic role of $\mathrm{Cx} 46$ in breast cancer [24]. Increased Cx26 and Cx43 levels have also been associated with breast cancer metastasis [22]. However, the prognostic and predictive role of connexins in relation to neoadjuvant chemotherapy has not been investigated before.

Gap junctions are thought to inhibit cell cycle though most findings relate to $\mathrm{Cx} 43$ [19]. Forced Cx43 expression can block G1/S phase transition or delay G2/M transition through increasing p $21^{\text {waf1 }}$ and reducing Cdk2 levels [37]. Also, Cx43 expression and gap junction coupling can be reduced through phosphorylation by Cdk1/cyclinB complex at G2/M transition [19]. Therefore, Cx expression may dynamically change during cell cycle. In breast cancer cell lines low levels of Cx26 at G1/S phase, increasing levels during late $\mathrm{S} / \mathrm{G} 2$ phase transition and downregulation in $\mathrm{M}$ phase was observed [38].

The correlations we have revealed between Cx expression and hormone receptor, HER2 or Ki67 levels suggest that connexin isotypes are differentially involved in the regulation of breast cancer cell functions. In concordance with published data we found a positive correlation between Cx43 expression and hormone receptor levels both before and after treatment in primary breast cancer [39]. However, either Cx43 or Cx26 protein can be elevated again in the lymph node metastasis of invasive breast cancer [22]. We also found a positive correlation between Cx32 and HER2 expression and between Cx32 and Ki67 expression; and a negative correlation between Cx43 and HER2 levels and between Cx46 and Ki67 expression. As expected, Ki67 expression showed a negative correlation with hormone receptor expression and a positive correlation with HER2 levels. These results may imply potential tumour suppressive functions for $\mathrm{Cx} 43$ and $\mathrm{Cx} 46$ in primary breast cancer as opposed to $\mathrm{Cx} 26$

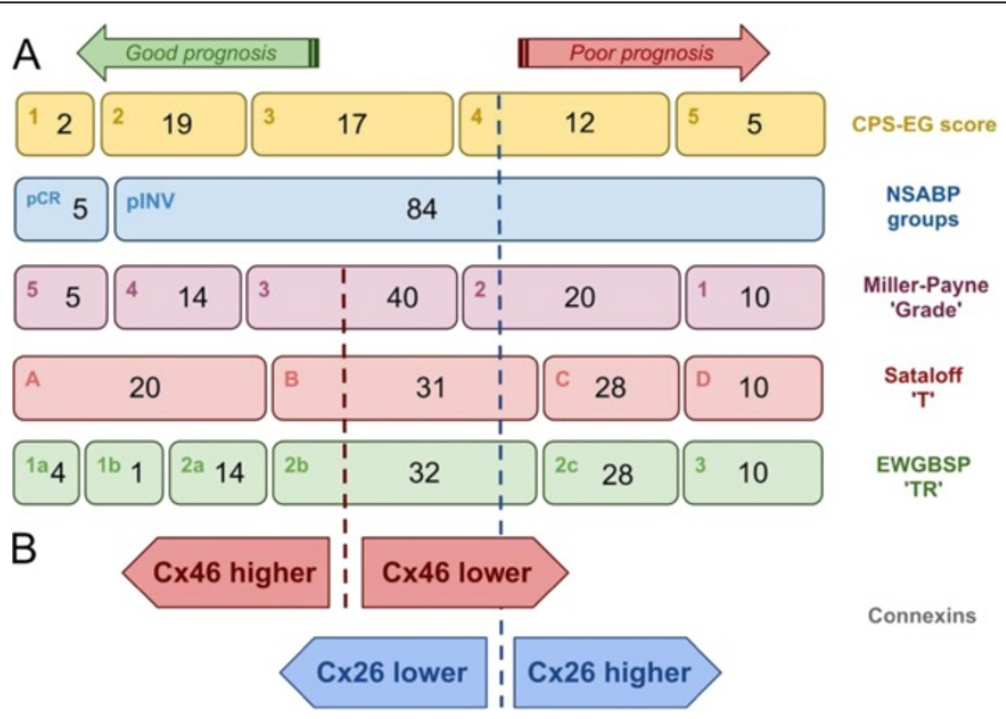

Figure 5 Distribution of the studied patient cohort within current classifications and the power of $\mathrm{Cx} 26$ and Cx46 expression to discriminate good and poor prognostic groups within the equivocal categories of NSABP, Miller-Payne G, Sataloff T, EWGBSP TR and MDAAC CPS-EG systems that are used for assessing response to neoadjuvant chemotherapy (A). Both high Cx46 levels and low Cx26 levels correlate with good prognosis (B). 


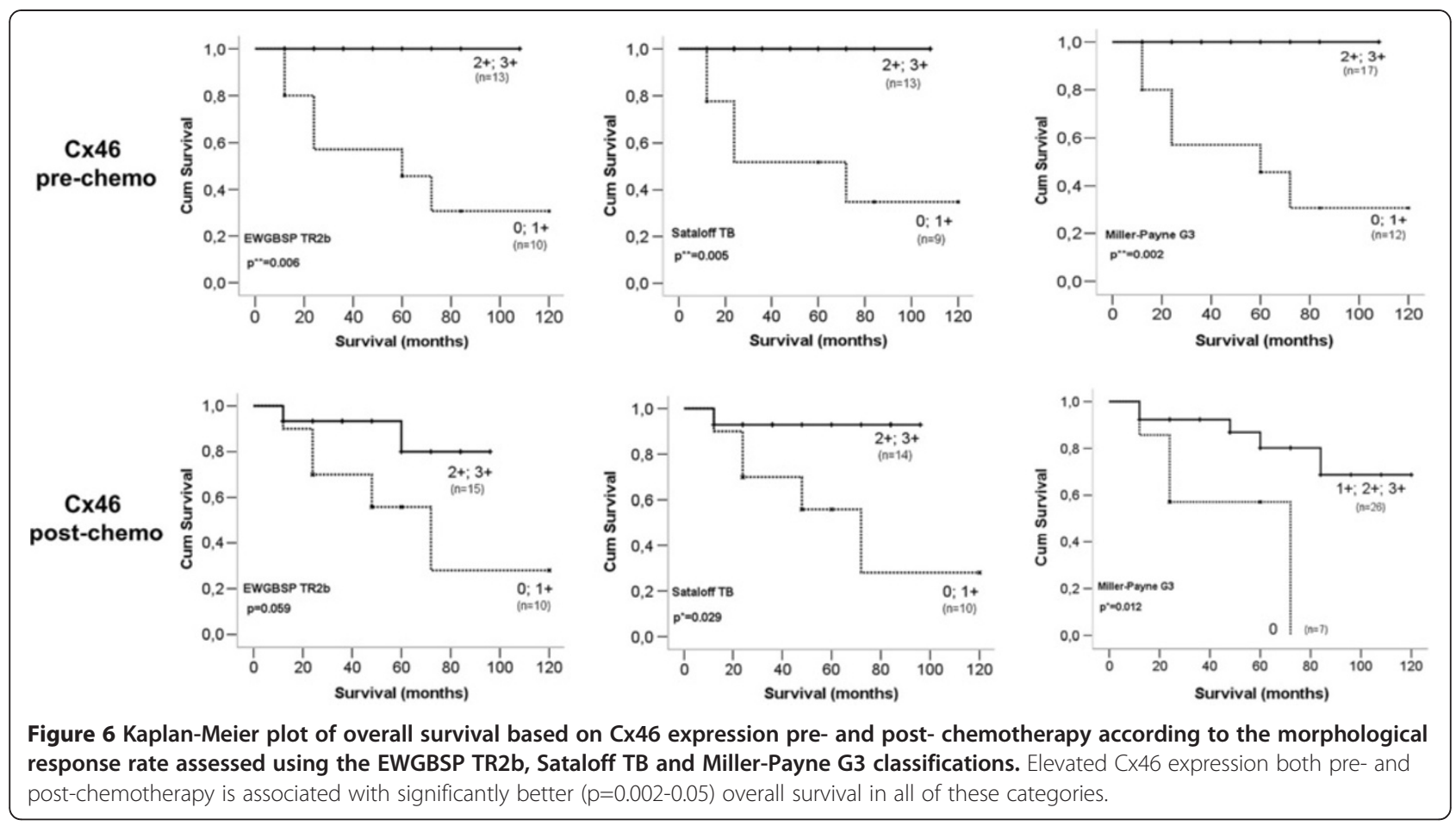

or Cx32 expression which may be tumour protective as well. The positive correlation between Cx46 levels and nodal status may be explained by the lack of correlation between nodal status and overall survival in our cohort and that the majority of our cases were ER positive.

In this study, reduced Cx26 and Cx32 levels and elevated Cx46 expression upon chemotherapy may also reflect the efficiency of chemotherapy. The exact role of connexins in carcinogenesis and metastasis formation is controversial or more probably context dependent.

Table 4 Multivariate Cox-regression analysis of connexin expression, hormone receptors and CPS-EG classification in breast cancer patient groups

\begin{tabular}{|c|c|c|c|c|c|}
\hline \multirow[t]{2}{*}{ Patient group } & \multirow[t]{2}{*}{ Parameters } & \multirow[t]{2}{*}{ P-value } & \multirow[t]{2}{*}{$H R$} & \multicolumn{2}{|c|}{$95 \% \mathrm{Cl}$} \\
\hline & & & & Lower & Upper \\
\hline \multirow[t]{5}{*}{ whole cohort } & Cx26 post & 0.303 & 0.450 & 0.098 & 2.055 \\
\hline & $E R_{\text {post }}$ & 0.050 & 2,739 & 0.999 & 7.504 \\
\hline & Cx26 post & 0.157 & 0.134 & 0.008 & 2.173 \\
\hline & $P R_{\text {post }}$ & 0.027 & 6.509 & 1.241 & 34.140 \\
\hline & CPS EG & 0.967 & 0.000 & 0.000 & $1.381 \mathrm{E} 26$ \\
\hline \multirow[t]{2}{*}{ EWGBSP TR2b } & Cx46 post & 0.790 & 4.400 & 0.844 & 22.950 \\
\hline & $\mathrm{PR}_{\text {post }}$ & 0.115 & 3.809 & 0.722 & 20.111 \\
\hline \multirow[t]{2}{*}{ Sataloff TB } & Cx46 post & 0.226 & 3.782 & 0.439 & 32.600 \\
\hline & $P R_{\text {post }}$ & 0.244 & 2.791 & 0.496 & 15.711 \\
\hline \multirow[t]{2}{*}{ Miller-Payne G3 } & Cx46 post & 0.104 & 3.322 & 0.782 & 14.105 \\
\hline & $P R_{\text {post }}$ & 0.075 & 4.400 & 0.861 & 22.481 \\
\hline
\end{tabular}

post: after chemotherapy; $H R$ : hazard ratio; Cl: confidence intervals.
Several previous studies addressed the changes of biomarker expression after primary systemic therapy in breast cancer [40-43]. Discordance in hormone receptor status has been reported to vary from $2.5 \%$ to $~ 50 \%$ [ 41 43]. The same has been found for HER2 status with altered immunophenotype found in up to $43 \%$ of cases especially when immunohistochemistry was the choice of assay [42,43]. Although not analysed in detail here, we found discordant ER/PR/HER2 status in only $<10 \%$ of our studied patients after neoadjuvant chemotherapy.

The predictive role of hormone receptors and HER2 status in view of partial and complete pathological response in breast cancer has also been extensively investigated [44-49]. HER2 positive, ER negative breast cancers with poor histological differentiation without nodal involvement have achieved better complete pathological response compared to those with lymph node metastasis [44,4650]. In our cohort, ER positive HER2 negative tumours dominated representing prognostically intermediate categories. Therefore, we did not correlate hormone and HER2 expression with partial and complete pathological response to neoadjuvant therapy.

We have classified clinicopathological parameters in breast cancers according to five current systems and could establish a significantly improved overall survival in cases scoring 1-2 against those scoring 3-5 only when using the CPS-EG classification. The lack of correlation with overall survival in the rest of classifications (NSABP, Miller-Payne, Sataloff and EWGBSP) is probably due to the fact that the majority of our cases fell 
into the prognostically intermediate categories. As we are aware, comprehensive assessment of therapy response using the major classifications as we tested here has not been done so far by others in breast cancer treated with neoadjuvant therapy.

\section{Conclusion}

Although our data are limited by the small sample size, they support additional studies of Cx26 and Cx46 to further refine outcome prediction for the intermediate groups as defined by currently used classification systems of pathological response to neoadjuvant chemotherapy in breast cancer.

\section{Competing interests}

The authors declare no conflict of interest concerning the content of this manuscript.

\section{Authors' contribution}

ZV initiated this study with TK. Clinicopathological data were retrieved by CR in co-operation with $\mathrm{CL}$ and BP.CR made the tissue microarray. HM helped in writing the manuscript. TK supervised immunomorphological studies performed by IT and compiled the paper together with ZV and IT. IT, MSZ and BW performed the statistics after scoring together with JK. JK also gave advice on the interpretation of the results. All authors read and approved the final manuscript.

\section{Acknowledgements}

The authors are indebted to Edit Parsch for technical assistance and to Martina Storz for tissue microarrays construction. This work was supported by MTA TKI of the Hungarian Academy of Sciences.

\section{Author details}

${ }^{1} 1$ st Department of Pathology \& Experimental Cancer Research, Semmelweis University, Budapest, Hungary. ${ }^{2}$ MTA-SE Tumour Progression Research Group, Budapest, Hungary. ${ }^{3}$ 2nd Department of Pathology, Semmelweis University, Budapest, Hungary. ${ }^{4}$ 2nd Department of Internal Medicine, Semmelweis University, Budapest, Hungary. ${ }^{5}$ Division of Gynecology, University Hospital Zurich, Zurich, Switzerland. ${ }^{6}$ Breast Cancer Center Seefeld, Zurich, Switzerland. ${ }^{7}$ Institute of Surgical Pathology, University Hospital Zurich, Zurich, Switzerland.

Received: 27 June 2012 Accepted: 30 January 2013

Published: 2 February 2013

\section{References}

1. Ferlay J, Shin HR, Bray F, Forman D, Mathers C, Parkin DM: Estimates of worldwide burden of cancer in 2008: GLOBOCAN 2008. Int J Cancer 2010, 127(12):2893-2917.

2. Payne SJ, Bowen RL, Jones $J \mathrm{~L}$, Wells $C A$ : Predictive markers in breast cancer-the present. Histopathology 2008, 52(1):82-90.

3. Cserni G, Francz M, Jaray B, Kalman E, Kovacs I, Kulka J, Orosz Z, Udvarhelyi $N$, Vass L: Pathologic diagnosis and histopathology record of breast cancer. Magy Onkol 2010, 54(3):217-226.

4. Kuroi $K$, Toi M, Tsuda $H$, Kurosumi M, Akiyama F: Issues in the assessment of the pathologic effect of primary systemic therapy for breast cancer. Breast Cancer 2006, 13(1):38-48.

5. Mittendorf EA, Jeruss JS, Tucker SL, Kolli A, Newman LA, Gonzalez-Angulo AM, Buchholz TA, Sahin AA, Cormier JN, Buzdar AU, et al: Validation of a novel staging system for disease-specific survival in patients with breast cancer treated with neoadjuvant chemotherapy. J Clin Oncol 2011 29(15):1956-1962.

6. Sahoo S, Lester SC: Pathology of breast carcinomas after neoadjuvant chemotherapy: an overview with recommendations on specimen processing and reporting. Arch Pathol Lab Med 2009, 133(4):633-642.

7. Shien T, Shimizu C, Seki K, Shibata T, Hojo T, Ando M, Kohno T, Katsumata N, Akashi-Tanaka S, Kinoshita T, et al: Comparison among different classification systems regarding the pathological response of preoperative chemotherapy in relation to the long-term outcome. Breast Cancer Res Treat 2009, 113(2):307-313.

8. Jeruss JS, Mittendorf EA, Tucker SL, Gonzalez-Angulo AM, Buchholz TA Sahin AA, Cormier JN, Buzdar AU, Hortobagyi GN, Hunt KK: Combined use of clinical and pathologic staging variables to define outcomes for breast cancer patients treated with neoadjuvant therapy. J Clin Oncol 2008, 26(2):246-252.

9. Jeruss JS, Mittendorf EA, Tucker SL, Gonzalez-Angulo AM, Buchholz TA, Sahin AA, Cormier JN, Buzdar AU, Hortobagyi GN, Hunt KK: Staging of breast cancer in the neoadjuvant setting. Cancer Res 2008, 68(16):6477-6481.

10. Czyz J: The stage-specific function of gap junctions during tumourigenesis. Cell Mol Biol Lett 2008, 13(1):92-102.

11. Goodenough DA, Paul DL: Gap junctions. Cold Spring Harb Perspect Biol 2009, 1(1):a002576.

12. Vinken M, Vanhaecke T, Papeleu P, Snykers S, Henkens T, Rogiers V: Connexins and their channels in cell growth and cell death. Cell Signal 2006, 18(5):592-600.

13. Rackauskas M, Neverauskas V, Skeberdis VA: Diversity and properties of connexin gap junction channels. Medicina (Kaunas) 2010, 46(1):1-12.

14. Houghton FD: Role of gap junctions during early embryo development Reproduction 2005, 129(2):129-135.

15. Krysko DV, Leybaert L, Vandenabeele P, D'Herde K: Gap junctions and the propagation of cell survival and cell death signals. Apoptosis 2005 10(3):459-469

16. Herve JC, Derangeon M, Sarrouilhe D, Giepmans BN, Bourmeyster N: Gap junctional channels are parts of multiprotein complexes. Biochim Biophys Acta 2012, 1818(8):1844-1865.

17. Locke D: Gap junctions in normal and neoplastic mammary gland. J Pathol 1998, 186(4):343-349.

18. Pointis G, Fiorini C, Gilleron J, Carette D, Segretain D: Connexins as precocious markers and molecular targets for chemical and pharmacological agents in carcinogenesis. Curr Med Chem 2007, 14(21):2288-2303.

19. Cronier L, Crespin S, Strale PO, Defamie N, Mesnil M: Gap junctions and cancer: new functions for an old story. Antioxid Redox Signal 2009, 11(2):323-338

20. Oviedo-Orta E, Errington RJ, Evans WH: Gap junction intercellular communication during lymphocyte transendothelial migration. Cell Biol Int 2002, 26(3):253-263.

21. Kanczuga-Koda L, Sulkowska M, Koda M, Rutkowski R, Sulkowski S: Increased expression of gap junction protein-connexin 32 in lymph node metastases of human ductal breast cancer. Folia Histochem Cytobiol 2007, 45(Suppl 1):S175-S180.

22. Kanczuga-Koda L, Sulkowski S, Lenczewski A, Koda M, Wincewicz A, Baltaziak M, Sulkowska M: Increased expression of connexins 26 and 43 in lymph node metastases of breast cancer. J Clin Pathol 2006, 59(4):429-433

23. McLachlan E, Shao Q, Laird DW: Connexins and gap junctions in mammary gland development and breast cancer progression. J Membr Biol 2007, 218(1-3):107-121.

24. Banerjee D, Gakhar G, Madgwick D, Hurt A, Takemoto D, Nguyen TA A novel role of gap junction connexin 46 protein to protect breast tumours from hypoxia. Int J Cancer 2010, 127(4):839-848.

25. Fattaneh A, Tavassoli PD: WHO Series, Tumours of the Breast and Female Genital Organs.; 2003:18-19.

26. Wolff AC, Hammond ME, Schwartz JN, Hagerty KL, Allred DC, Cote RJ, Dowsett M, Fitzgibbons PL, Hanna WM, Langer A, et al: American Society of Clinical Oncology/College of American Pathologists guideline recommendations for human epidermal growth factor receptor 2 testing in breast cancer. J Clin Oncol 2007, 25(1):118-145.

27. Kononen J, Bubendorf L, Kallioniemi A, Barlund M, Schraml P, Leighton S, Torhorst J, Mihatsch MJ, Sauter G, Kallioniemi OP: Tissue microarrays for high-throughput molecular profiling of tumour specimens. Nat Med 1998, 4(7):844-847

28. Theurillat JP, Zurrer-Hardi U, Varga Z, Barghorn A, Saller E, Frei C, Storz M, Behnke S, Seifert B, Fehr M, et al: Distinct expression patterns of the immunogenic differentiation antigen NY-BR-1 in normal breast, testis and their malignant counterparts. Int J Cancer 2008, 122(7):1585-1591.

29. Sohl G, Willecke K: Gap junctions and the connexin protein family. Cardiovasc Res 2004, 62(2):228-232. 
30. Krenacs T, Ficsor L, Varga SV, Angeli V, Molnar B: Digital microscopy for boosting database integration and analysis in TMA studies. Methods in molecular biology (Clifton, NJ) 2010, 664:163-175.

31. Miglietta L, Vanella P, Canobbio L, Naso C, Cerisola N, Meszaros P, Parodi MA, Morabito F: Prognostic value of estrogen receptor and Ki-67 index after neoadjuvant chemotherapy in locally advanced breast cancer expressing high levels of proliferation at diagnosis. Oncology 2010, 79(3-4):255-261.

32. Kyo N, Yamamoto H, Takeda Y, Ezumi K, Ngan CY, Terayama M, Miyake M, Takemasa I, Ikeda M, Doki Y, et al: Overexpression of connexin 26 in carcinoma of the pancreas. Oncol Rep 2008, 19(3):627-631.

33. Tate AW, Lung T, Radhakrishnan A, Lim SD, Lin X, Edlund M: Changes in gap junctional connexin isoforms during prostate cancer progression. Prostate 2006, 66(1):19-31.

34. Liu X, Furuya T, Li D, Xu J, Cao X, Li Q, Xu Z, Sasaki K: Connexin 26 expression correlates with less aggressive phenotype of intestinal typegastric carcinomas. Int J Mol Med 2010, 25(5):709-716.

35. Nomura S, Maeda K, Noda E, Inoue T, Fukunaga S, Nagahara H, Hirakawa K. Clinical significance of the expression of connexin26 in colorectal cancer. J Exp Clin Cancer Res 2010, 29:79.

36. Bier A, Oviedo-Landaverde I, Zhao J, Mamane Y, Kandouz M, Batist G: Connexin43 pseudogene in breast cancer cells offers a novel therapeutic target. Mol Cancer Ther 2009, 8(4):786-793.

37. Kamei J, Toyofuku T, Hori M: Negative regulation of $\mathrm{p} 21$ by beta-catenin/TCF signaling: a novel mechanism by which cell adhesion molecules regulate cell proliferation. Biochem Biophys Res Commun 2003, 312(2):380-387.

38. Lee SW, Tomasetto C, Paul D, Keyomarsi K, Sager R: Transcriptional downregulation of gap-junction proteins blocks junctional communication in human mammary tumour cell lines. J Cell Biol 1992 118(5):1213-1221.

39. Conklin C, Huntsman D, Yorida E, Makretsov N, Turbin D, Bechberger JF, Sin WC, Naus CC: Tissue microarray analysis of connexin expression and its prognostic significance in human breast cancer. Cancer Lett 2007, 255(2):284-294.

40. Kulka J, Tokes AM, Toth Al, Szasz AM, Farkas A, Borka K, Jaray B, Szekely E, Istok R, Lotz G, et al: Immunohistochemical phenotype of breast carcinomas predicts the effectiveness of primary systemic therapy. Magy Onkol 2009, 53(4):335-343.

41. Neubauer H, Gall C, Vogel U, Hornung R, Wallwiener D, Solomayer E, Fehm $\mathrm{T}$ : Changes in tumour biological markers during primary systemic chemotherapy (PST). Anticancer Res 2008, 28(3B):1797-1804.

42. van de Ven S, Smit VT, Dekker TJ, Nortier JW, Kroep JR: Discordances in ER, PR and HER2 receptors after neoadjuvant chemotherapy in breast cancer. Cancer Treat Rev 2011, 37(6):422-430.

43. Varga Z, Caduff R, Pestalozzi B: Stability of the HER2 gene after primary chemotherapy in advanced breast cancer. Virchows Arch 2005, 446(2):136-141.

44. Darb-Esfahani S, Loibl S, Muller BM, Roller M, Denkert C, Komor M, Schluns K, Blohmer JU, Budczies J, Gerber B, et al: Identification of biology-based breast cancer types with distinct predictive and prognostic features: role of steroid hormone and HER2 receptor expression in patients treated with neoadjuvant anthracycline/taxane-based chemotherapy. Breast Cancer Res 2009, 11(5):R69.

45. Denkert C, Sinn BV, Issa Y, Maria Muller B, Maisch A, Untch M, von Minckwitz G, Loibl S: Prediction of Response to Neoadjuvant Chemotherapy: New Biomarker Approaches and Concepts. Breast Care (Basel) 2011, 6(4):265-272.

46. Guiu S, Gauthier M, Coudert B, Bonnetain F, Favier L, Ladoire S, Tixier H, Guiu B, Penault-Llorca F, Ettore F, et al: Pathological complete response and survival according to the level of HER-2 amplification after trastuzumab-based neoadjuvant therapy for breast cancer. $\mathrm{Br} J$ Cancer 2010, 103(9):1335-1342.

47. Precht LM, Lowe KA, Atwood M, Beatty JD: Neoadjuvant chemotherapy of breast cancer: tumour markers as predictors of pathologic response, recurrence, and survival. Breast J 2010, 16(4):362-368.

48. Untch M, Fasching PA, Konecny GE, Hasmuller S, Lebeau A, Kreienberg $\mathrm{R}$, Camara O, Muller V, du Bois A, Kuhn T, et al: Pathologic complete response after neoadjuvant chemotherapy plus trastuzumab predicts favorable survival in human epidermal growth factor receptor 2-overexpressing breast cancer: results from the TECHNO trial of the AGO and GBG study groups. J Clin Oncol 2011, 29(25):3351-3357.
49. von Minckwitz G, Sinn HP, Raab G, Loibl S, Blohmer JU, Eidtmann H, Hilfrich J, Merkle E, Jackisch C, Costa SD, et al: Clinical response after two cycles compared to HER2, Ki-67, p53, and bcl-2 in independently predicting a pathological complete response after preoperative chemotherapy in patients with operable carcinoma of the breast. Breast Cancer Res 2008, 10(2):R30.

50. Osako T, Horii R, Matsuura M, Ogiya A, Domoto K, Miyagi Y, Takahashi S, Ito $Y$, Iwase T, Akiyama F: Common and discriminative clinicopathological features between breast cancers with pathological complete response or progressive disease in response to neoadjuvant chemotherapy. J Cancer Res Clin Oncol 2010, 136(2):233-241.

doi:10.1186/1471-2407-13-50

Cite this article as: Teleki et al:: The potential prognostic value of connexin 26 and 46 expression in neoadjuvant-treated breast cancer. BMC Cancer 2013 13:50.

\section{Submit your next manuscript to BioMed Central and take full advantage of:}

- Convenient online submission

- Thorough peer review

- No space constraints or color figure charges

- Immediate publication on acceptance

- Inclusion in PubMed, CAS, Scopus and Google Scholar

- Research which is freely available for redistribution 